\title{
Transcriptomic changes arising during light-induced sporulation in Physarum polycephalum
}

Israel Barrantes ${ }^{1,4,6}$, Gernot Glockner $2,3,5$, Sonja Meyer ${ }^{4,6}$, Wolfgang Marwan ${ }^{4,6 *}$

\begin{abstract}
Background: Physarum polycephalum is a free-living amoebozoan protist displaying a complex life cycle, including alternation between single- and multinucleate stages through sporulation, a simple form of cell differentiation. Sporulation in Physarum can be experimentally induced by several external factors, and Physarum displays many biochemical features typical for metazoan cells, including metazoan-type signaling pathways, which makes this organism a model to study cell cycle, cell differentiation and cellular reprogramming.

Results: In order to identify the genes associated to the light-induced sporulation in Physarum, especially those related to signal transduction, we isolated RNA before and after photoinduction from sporulation- competent cells, and used these RNAs to synthesize cDNAs, which were then analyzed using the 454 sequencing technology. We obtained 16,669 CDNAs that were annotated at every computational level. 13,169 transcripts included hit count data, from which 2,772 displayed significant differential expression (upregulated: 1,623; downregulated: 1,149). Transcripts with valid annotations and significant differential expression were later integrated into putative networks using interaction information from orthologs.

Conclusions: Gene ontology analysis suggested that most significantly downregulated genes are linked to DNA repair, cell division, inhibition of cell migration, and calcium release, while highly upregulated genes were involved in cell death, cell polarization, maintenance of integrity, and differentiation. In addition, cell death- associated transcripts were overrepresented between the upregulated transcripts. These changes are associated to a network of actin-binding proteins encoded by genes that are differentially regulated before and after light induction.
\end{abstract}

\section{Background}

Physarum polycephalum, commonly known as "slime mold", belongs to the mycetozoan group of Amoebozoa. Physarum follows a complex life cycle with haploid and diploid cell types, formed in temporal order as triggered by environmental stimuli [1]. The plasmodium is a multinucleate single cell whose nuclei display natural synchrony with respect to cell cycle and differentiation status. During several days of starvation, a plasmodium grown to macroscopic size becomes competent to sporulate. Sporulation can then be triggered experimentally by exposing a competent plasmodium to a pulse of blue or far-red light, or to heat shock. As plasmodial nuclei

\footnotetext{
* Correspondence: marwan@mpi-magdeburg.mpg.de

${ }^{4}$ Max Planck Institute for Dynamics of Complex Technical Systems and Magdeburg Centre for Systems Biology (MaCS), Otto von Guericke University, Magdeburg, Germany
}

(c) 2010 Barrantes et al; licensee BioMed Central Ltd. This is an Open Access article distributed under the terms of the Creative Commons Attribution License (http://creativecommons.org/licenses/by/2.0), which permits unrestricted use, distribution, and reproduction in any medium, provided the original work is properly cited. are synchronous, and because the timing of the differentiation program in individual plasmodia can be reproduced experimentally, the stage-specific gene expression program that leads to sporulation can be analyzed at high resolution [2-4]. Recent large scale cDNA surveys $[5,6]$ provide a basis for studying the phenomenon at the transcriptomic level.

In order to identify the differentially expressed genes associated with the commitment to sporulation, we characterized and compared two cDNA libraries prepared from competent and light-induced plasmodia using massive parallel sequencing technology [7]. We employed this method because it does not rely on reference transcripts for quantitation, previous cloning steps are not required, it does not have an upper limit for quantitation, and it is a relatively unbiased approach [8]. From the comparison of annotations and transcript 
quantitations, we found that most differentially expressed genes encode proteins associated to a network of actin-binding proteins. Components of this putative interaction network are associated to development, DNA repair, cell division, calcium release, cell death, and maintenance of cell integrity.

\section{Results}

\section{Sequencing and Profiling of cDNAs expressed in} competent and light-induced plasmodia

Separate cDNA libraries were constructed from polyA ${ }^{+}$ RNA isolated from two sources: $(i)$ competent plasmodia; and (ii) sporulation- induced plasmodia (competent plasmodia harvested six hours after exposure to far-red light). The cDNAs libraries were then analyzed using massive parallel sequencing $[7,8]$. Transcripts were annotated at every bioinformatic level, and the annotation data was used to infer hypothetical interaction networks from differentially regulated genes. The whole approach is summarized in the [Additional File 1: Figure S1].

From the pyrosequencing, we obtained a total output of $61.9 \mathrm{Mb}$ from two runs, corresponding to the starved (26.1 Mb) and light-induced (35.8 Mb) plasmodia libraries. Considering that Physarum possess a $300 \mathrm{Mb}$ genome, and assuming that $10 \%$ is encoding genes, we estimate a $2.06 \times$ coverage of protein coding sequences. The assembled sequencing output consisted of 26,037 sequences, and large cDNAs from this assembly $(>500$ bp) were then joined to a previously available sequence dataset [6], to form a comprehensive set of representative transcripts. This analysis produced 16,669 sequences, 13,169 of these containing transcript abundance data: 125,456 reads from competent and 99,632 reads from light-induced plasmodia, respectively [Additional File 2]. We used this abundance data (number of reads for each assembled transcript) as a measure of expression, which we defined as "hit counts". The remaining contigs without hit counts consisted of previously sequenced clones from a normalized cDNA library prepared from competent plasmodia [6], indicating that the normalization produced a broader coverage of transcripts. From 11,399 cDNA contigs detected in the competent plasmodia library $(10,689$ in lightinduced plasmodia), over 4,227 were represented with at least five hits (3,553 in light-induced plasmodia [Additional File 3: Figure S2]). Conversely, 8,711 transcripts $(52,3 \%)$ were found with 5 or less sequence hits in both samples. For statistical reasons, no statement on the differential expression from this fraction could be made. Between contigs with lowest hit counts, 2,437 cDNA species were represented by just one hit (competent plasmodia), and 2,621 from light-induced sample [Additional File 3: Figure S2].
We compared the transcript hit counts between different libraries as a measure of differential gene expression. As most contig species were represented by low hit counts, we normalized the number of hits. To this end, we first obtained the relative frequency (number of hits divided by the total hits on a given condition), and later we calculated the relative frequencies for each contig in the two cDNA samples compared to each other. Given that each EST belongs to a single gene, the significance of its differential expression depends only on the number of hits, respect to the total number of hits on each library [9]. Following these assumptions, we found 2,772 cDNAs that displayed significant differential expression $(P$-value $<0.05)$. All contig species, regardless of whether differentially expressed or not were submitted to the Sequence Read Archive subset of GenBank [10] (Accession numbers SRX012830 and SRX012831).

The newly assembled contigs were compared against sequence databases using BLASTX [11]. This analysis revealed that 3,310 sequences have significant similarity $(\leq 1 \times 10-15)$ to existing sequences in SwissProt [12], 3,651 to the protozoa subset from RefSeq [13], and 3,345 to proteins of the related model organism Dictyostelium discoideum (dictyBase; [14]). From the 13,169 sequences with hit counts data, orthologs were identified for 5,544 transcripts $(1,287$ of these with significant differential expression; [Additional File 4]).

Later, in order to identify differentially regulated genes, we clustered the contig species into expression groups according to their relative frequencies in both conditions. As a result, we found contigs encoding orthologs related to cell division (meiosis-related protein MEI2; DNA polymerase beta; actin) and protein synthesis and degradation (elongation factor 1- alpha; cathepsin-L cysteine protease) with higher relative frequencies in the competent plasmodial library. Similarly, we found orthologs related to the cytoskeleton (spire; actophorin; cell wall integrity and stress response component, WSC1) and cell differentiation genes (CudA) with greater relative frequencies in the light- induction library (Figure 1; [Additional File 5: Table S1]).

\section{Gene Ontology Annotation of the Transcriptome}

The Gene Ontology (GO) project [15] is an annotation framework that provides a standardized vocabulary that is used to assign function to uncharacterized sequences, based on three main categories: biological processes (BP), molecular functions (MF) and cellular components (CC). We employed BLAST2GO [16], a tool that associates GO terms to sequences based in several annotation evidences, to classify gene function in our dataset. Using the BLASTX hits (annotation e-value cutoff $<1 \times 10-6$ ), together with GO terms previously extracted from 


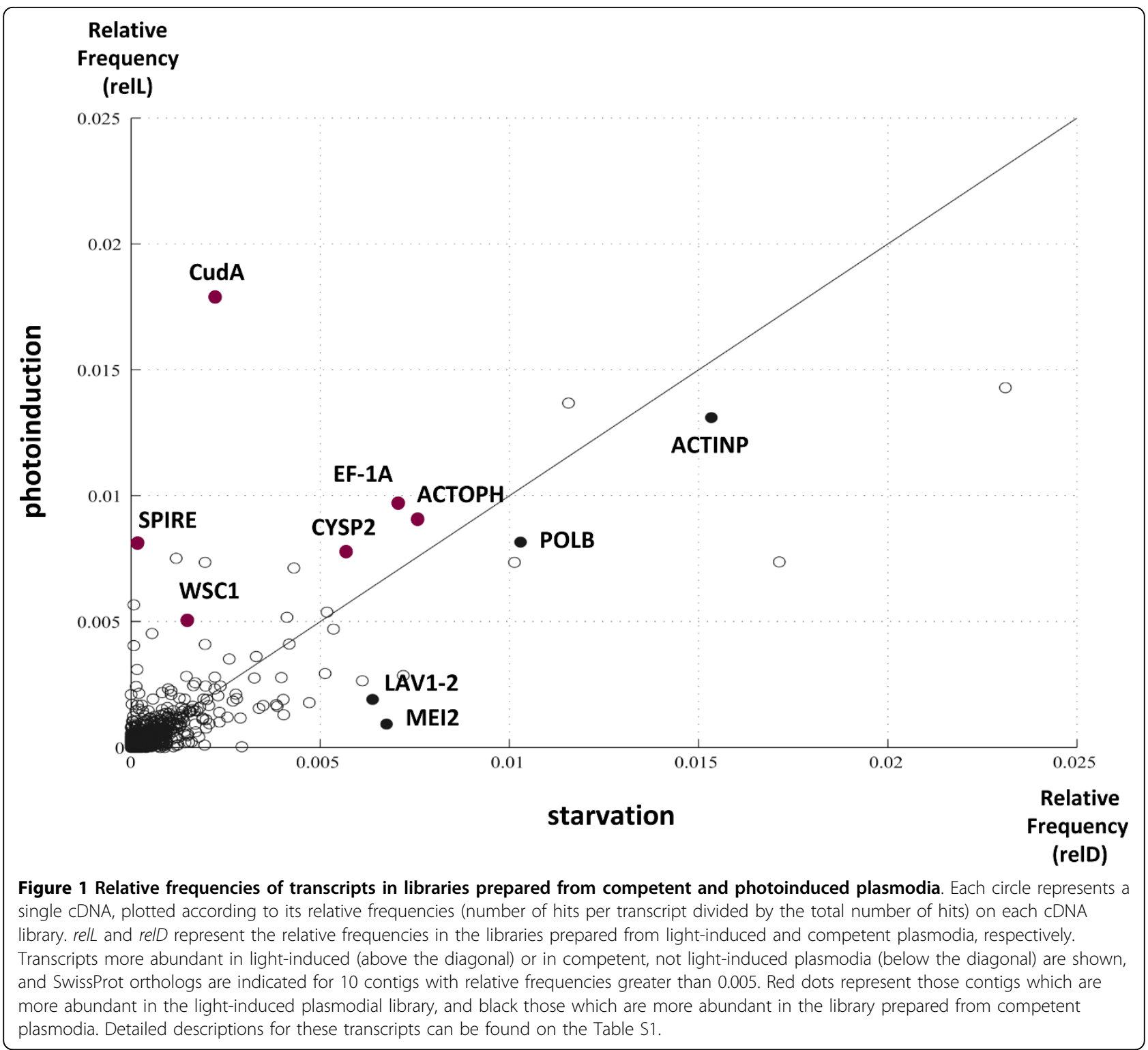

InterPro domain searches [17], we inferred 13,068 GO annotations for 3,304 (20\%) cDNAs, with 11,446 annotations belonging to 2,459 sequences with hit counts data. Transcripts were associated to biological processes $(\mathrm{n}=$ $2,437 ; 15 \%)$, molecular functions $(\mathrm{n}=2,801 ; 17 \%)$, and cellular components $(\mathrm{n}=2,023 ; 12 \%)$. As many as 2,136 (13\%), 1,663 (10\%) and 1,645 (10\%) sequences were annotated with a combination of MF and BP terms, MF and $\mathrm{CC}$, and $\mathrm{BP}$ and $\mathrm{CC}$ terms respectively, and 1,487 cDNAs were annotated with MF, BP and CC terms altogether. Later, in order to analyze the differences between the two condition groups with respect to the GO annotations, we carried out Fisher exact tests using the Gossip module [18] from BLAST2GO. We found that the 'cell development' (GO:0048468), 'cell death'
(GO:0008219) and 'death' (GO:0016265) GO terms were overrepresented in cDNAs with higher relative frequencies in light-induced plasmodia (false discovery rate < 0.01 ), as compared to competent plasmodia [Additional File 6: Table S2].

\section{Pathway classification of transcripts}

Functional annotation can also be classified using the pathway-based definition of ortholog genes from the Kyoto Encyclopedia of Genes and Genomes (KEGG) database [19]. In order to categorize the transcripts in KEGG pathways, we employed the KAAS server [20], a tool that uses similarity information to assign a sequence to a KEGG ortholog (KO) identifier, with default parameters for ESTs. We mapped 2,716 (16\%) 
transcripts to 114 reference metabolic pathways, 1,904 including hit counts data, from which 770 correspond to cDNAs with higher relative frequencies in the library prepared from competent plasmodia, and 743 cDNAs in the library prepared from light-induced plasmodia respectively. In addition, 496 sequences in total were assigned to the KEGG BRITE hierarchies. Transcripts associated to the nucleotide metabolism $(\mathrm{n}=110)$ and citrate cycle $(n=40)$ had the highest representation for the reference metabolic pathways, and the Wnt, TGFbeta and Jak- STAT signaling pathways were also depicted for the whole dataset $(n=49,42$ and 32 matches respectively). In the whole dataset we identified 420 cDNAs with potential roles in cell differentiation, with molecular entities associated to kinases $(n=140)$ and GTP binding $(\mathrm{n}=110)$ having the highest representation in the BRITE hierarchies. In addition, we mapped 1,159 total enzyme commission (EC) numbers (418 unique) with 380 unique enzyme names in 851 transcripts, using the EC-module of BLAST2GO [16]. Later, in order to assess the global metabolic changes that occur after light induction, transcripts with KO identifiers were mapped using the KEGG Atlas tool [21]. For transcripts with higher relative frequencies in the competent plasmodia library, we mapped enzymes for the lipid biosynthesis (map00061) and oxidative phosphorylation (map00190) pathways. Conversely, we identified enzymes for the N-glycan biosynthesis (map00510), urea cycle (map00220) and fatty acid metabolism (map00071) pathways in transcripts with higher relative frequencies in the light-induced plasmodial library (Figure 2). In the end, we obtained 2,567 contigs annotated for GO terms, KEGG orthologs, and InterPro hits together [Additional File 7]. A summary of sequencing annotations and statistics is listed on the Table 1.

\section{Inference of Interaction Networks}

In order to identify the functional relationships between the annotated cDNAs, we searched for known interactions in the literature. First, we used the cDNAs that were previously clustered according to their relative frequencies (Figure 1; [Additional File 5: Table S1]), and included additional proteins whose interactions have been observed in the literature for Physarum. Using the "guilt by association" heuristic to link coexpressed transcripts into functional groups $[22,23]$, we inferred an interaction network between those transcripts. This network is primarily based on actin-binding activities (Figure 3).

Later, to identify genes with similar regulation, we listed those transcripts with highest rates of relative frequencies, counted in both cDNA libraries ([Additional File 8: Table S3]; [Additional File 9: Table S4]). As most of these highly differentially regulated transcripts did not show any sequence similarity to previously annotated genes, we clustered the subset of cDNAs with similarity to annotated genes according to two parameters: $(i)$ their rate of relative frequencies; and (ii) their statistical significance of differential expression [9]. In this way we listed those 20 transcripts with annotations that were most up- or most downregulated in light-induced plasmodia, based on the statistical significance of their differential expression $(\mathrm{P}<0.05$; Tables 2 and 3). Despite the apparent diversity in biochemical functions, we searched for known interactions between these two groups of transcripts.

From annotations of the top up- and down-regulated transcripts (Tables 2 and 3), and including the transcripts from the above mentioned analysis (Figure 3), we extended the initial putative network using Cytoprophet [24]. This Cytoscape [25] plugin predicts networks based on information from interaction databases, associated to Swiss Prot matches of newly annotated genes [26]. Accordingly, we found that most of these genes encoded proteins predicted to interact in a network of actin-binding proteins (coaA, ABP120, actobindin, FRGP, AFK, PROP; Figure 4). These genes encoding proteins orthologs of which are associated to cell division (MEI2, PUM2, CDC16), DNA repair (POLB, FEN1), signal transduction (PP2C, CDC16), and calcium-binding (LAV1-2, KCNIP2, GAD) are downregulated in light-induced plasmodia (Table 2; [Additional File 5: Table S1]). In turn, a different group of developmentally regulated genes is preferentially expressed after photoinduction, including genes the products of which are involved in signaling (DCR2, RGS2, YPTC6, pakA), protein processing (FKBP70, sequestosome-1, PSMA7, RR7), cell integrity (WSC1, CDC31), calcium-binding (MLR1, TRHY, PAT1), and developmentally regulated actin-binding, such as the elongation factor $1 \alpha$ (EF1A), spire, and actophorin (Table 3; [Additional File 5: Table S1]; Figures 3 and 4).

\section{Discussion}

The development of plasmodia competent for sporulation includes growth arrest, condensation of cellular constituents, and mitosis [27]. Sporulation of competent plasmodia can be triggered by a light pulse. Some proteins associated with the light-induced pathways that lead to sporulation have been described [2-4], suggesting that several signaling mechanisms are involved, but there are no studies that describe changes at the level of the whole transcriptome. In the present study we identified the most up- and downregulated transcripts, which are associated to a network of putative interactions (Figure 4). The network is hypothetical as interactions used for inference are based on data obtained from different organisms. For the sake of simplicity, the discussion will 


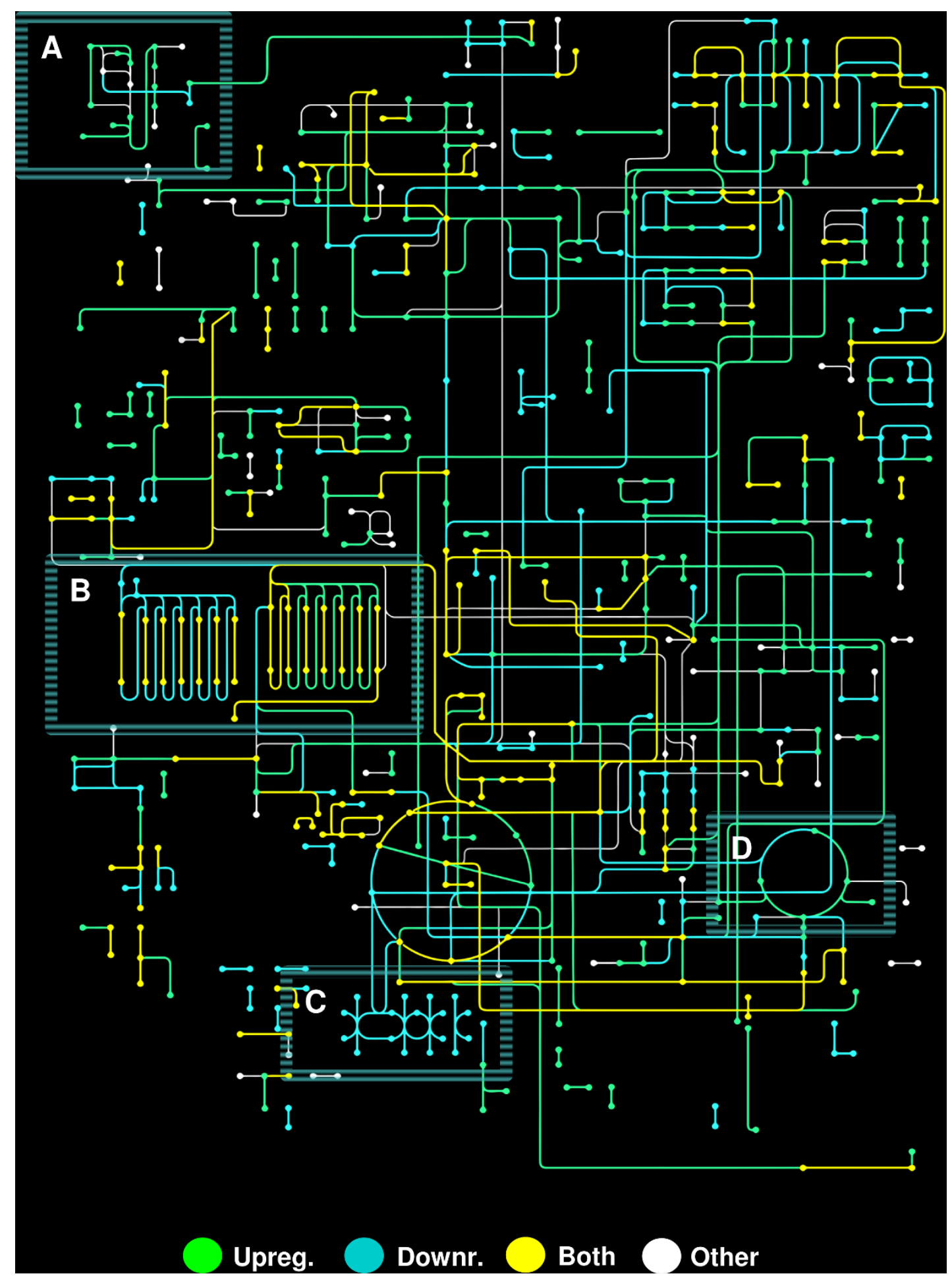

Figure 2 Metabolic Atlas of Physarum polycephalum. All P. polycephalum cDNAs (Refs [5,6] and our results) were sent for KEGG Ortholog [19] (KO) prediction using the KAAS server [20]. The output list of orthologs was used to plot this atlas with the KEGG mapping tool [21]. Nodes represent metabolites and edges (lines) correspond to enzymatic reactions. Colors are assigned to either down- (light blue) or upregulated (green) transcripts. Transcripts with equivalent relative frequencies in both novel cDNA libraries (relL/relD $=1$ ) are also depicted (yellow); white represent those cDNAs with no expression data. After photoinduction, most enzymes from the N-glycan biosynthesis (A) and the urea cycle (D) pathways are upregulated. In contrast, cDNAs mapped to the oxidative phosphorylation (C) had higher relative frequencies in competent plasmodia, whereas a change from fatty acid synthesis to degradation is seen after photoinduction (B). 
Table 1 Summary of the transcriptome sequencing and annotation

\begin{tabular}{lr}
\hline Sequencing & \\
\hline Total 454 reads & 405,363 \\
\hline Total sequencing output (Mb) & 125,456 \\
\hline Reads from the competent plasmodia library & 99,632 \\
\hline Reads from the light-induced plasmodia library & 16,669 \\
\hline contigs & 13,169 \\
\hline Total contigs & 2,103 \\
\hline Contigs with hit counts & $3,947^{\mathrm{a}}$ \\
\hline Contigs with at least 5 hits in both libraries & $4,972^{\mathrm{b}}$ \\
\hline More abundant in the library from competent plasmodia & 1,149 \\
\hline More abundant in the library from light-induced plasmodia & 1,623 \\
\hline Downregulated, significant differential expression & \\
\hline Upregulated, significant differential expression & 7,778 \\
\hline similarity search & 5,544 \\
\hline Total contigs with blastx results (e-value < 1E-3) & 1,287 \\
\hline Contigs with blastx results and hit counts & \\
\hline Contigs with blastx results and significant differential &
\end{tabular}

expression

annotations

\begin{tabular}{ll}
\hline Total contigs with GO annotations & 3,304 \\
\hline
\end{tabular}

\begin{tabular}{lr}
\hline Total contigs with KEGG orthologs & 2,716 \\
\hline
\end{tabular}

\begin{tabular}{ll}
\hline Total contigs with InterPro results & 6,813 \\
\hline
\end{tabular}

\begin{tabular}{lr}
\hline Contigs with GO annotations and hit counts & 2,459 \\
\hline
\end{tabular}

\begin{tabular}{ll}
\hline Contigs with KEGG orthologs and hit counts & 1,904 \\
\hline Contigs whe
\end{tabular}

Contigs with InterPro results and hit counts $\quad 5,180$

${ }^{a}$ Contigs with relative frequencies higher in the competent plasmodial library $($ relD/rell $>1)$, are classified as downregulated. Relative frequencies correspond to the number of hits per transcript divided by the total number of hits for each condition, competent (relD) and light-induced (relL) plasmodial libraries. ${ }^{b}$ Upregulated transcripts: Those with relative frequencies higher in the light-induced plasmodial library $($ rell/relD $>1)$

be focused on genes with predicted significant interactions $(\mathrm{P}>0.9)$.

\section{A network of actin-binding proteins is associated to changes during light-induced sporulation in Physarum}

The actin cytoskeleton of Physarum is essential for locomotion, division, and other biological processes [27]. Assembly and disassembly of actin filaments is controlled by a group of actin-binding proteins, whose activities in turn are regulated by specific signaling pathways. Physarum cell types differ in actin organization but express the same actin genes, suggesting that changes in actin-binding proteins are responsible for the differences in actin organization [28]. Physarum possesses several classes of actin-binding proteins, and most of these proteins display cell type-specific patterns of expression, but their precise roles are not known $[29,30]$. Nevertheless, expression changes in genes coding for actin-binding proteins correlate with modifications in cell organization and behavior [27]. In the present study, we found that some actin-binding genes were linked specifically to stages before and after photoinduction in the starved Physarum plasmodium.

Specifically, we identified protist orthologs for actinbinding proteins, including Dictyostelium coaA (Coactosin A [31]) and ABP-120 (actin-binding protein 120), and actobindin from Acanthamoeba, which binds actin monomers (Table 2) [32]. Coactosin A interferes with the capping of F-actin filaments [33], and is differentially expressed after metal exposure in worms [34]. ABP-120 organizes filamentous actin into networks of fibers, and Dictyostelium cells lacking ABP-120 have a severe phototaxis defect at the multicellular slug stage [35]. In addition, we noticed that transcripts coding for Physarum plasmodia-specific actin-binding proteins, such as profilin P (PROP) [30] and fragmin P (FRGP) [36], are downregulated after photoinduction (Figure 3). FRGP enables actin phosphorylation by the actin-fragmin kinase (AFK), and binds phosphorylated actin [29,36]. Therefore it is possible that during sporulation these proteins are involved in the reorganization of the subcellular compartments via interactions with the actin cytoskeleton.

\section{Transcripts linked to cell division and DNA repair are downregulated in the light-induced plasmodium}

After several days of starvation, cell processes must be limited in order to save energy. Coordination of several biological processes is then required, and thus regulation of these phenomena needs a pleiotropic transducer like the cAMP, which targets several signaling pathways, including those that limit cell proliferation [37]. Cell differentiation pathways regulated by cAMP levels have been described in Dictyostelium, a closely related protist [38]. For Physarum, we found that MEI2, a transcript controlled via cAMP levels, is downregulated in the light-induced plasmodium (Figure 3; [Additional File 5: Table S1]). MEI2 is an RNA-binding protein that encodes a CAMP-regulated positive regulator of meiosis in the yeast S.pombe $[39,40]$. This gene product is functionally related to the actin cytoskeleton via the cAMPdependent protein kinase A (PKA) $[37,38]$. Other transcripts downregulated in light-induced plasmodia associated to cell division and DNA repair comprised FEN1, CDC16 and PUM2. First, the Flap endonuclease 1 (FEN1) appears in several processes linked to the maintenance of the genome integrity, such as the UVinduced DNA repair [41], as well as in DNA replication and DNA recombination [42]. Second, the yeast cell division control protein 16 (CDC16), constitutes the catalytic subunit of the spg1p GTPase-activating protein, that is involved in the signal transduction controlling septum formation. CDC16 is involved in cytokinesis [43] 


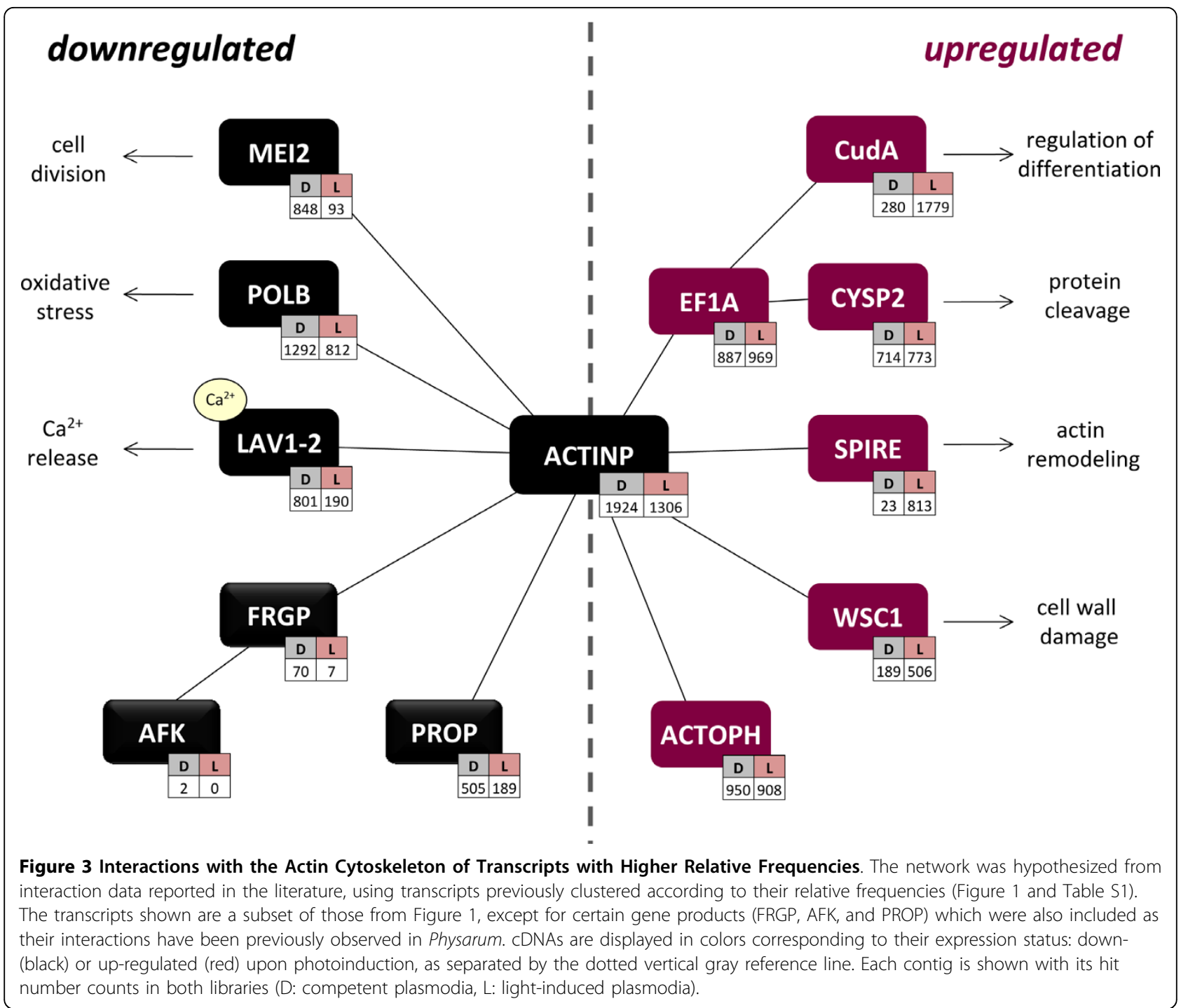

and is essential for proliferation, as spores lacking a functional CDC16 gene complete mitosis without undergoing cell cleavage [44]. Finally, PUM2 (Pumilio 2) encodes a RNA-binding protein associated to the control of meiosis during development [45]. Consequently, starvation seems to be the signal that regulates cell division while protecting the cells from oxidative stress, through cAMP-regulated pathways (Figure 3).

Other downregulated transcripts in the light-induced plasmodium comprised orthologs of transducers, such as FBL5, a leucine-repeat protein linked to phosphorylation-dependent ubiquitination [46], PARP1, an Oryza poly ADP-ribose polymerase, a phospholipase D from Phytophtora (PLD1), and the Arabidopsis phosphatase 2C (PP2C, also known as Poltergeist). In plants, G-proteins are involved in phospholipase $\mathrm{D}$ activation, and this also seems to be the case for Phytophtora [47]; on the other hand, PP2C operates in several signaling pathways that regulate stem cell differentiation [48]. It is then reasonable to consider that the differential expression of these transducers is also associated with the control of signaling mechanisms for differentiation, but more profound studies are needed to establish precise causal relationships.

\section{Calcium- binding proteins exhibit diverse regulation patterns in the light-induced plasmodium}

Transcripts identified as calcium-binding proteins displayed different patterns of expression regulation. These were either down- (LAV1-2, KCNIP2 and GAD) or upregulated (MLR1, TRHY, and PAT1) after light induction. LAV1-2 is a plasmodium-specific RNA of unknown function that encodes a protein containing an EF-hand type domain whose calcium-binding activity has been observed in vitro in Physarum [49]. LAV1-2 seems to act as a sensor of cell damage, releasing $\mathrm{Ca}^{2+}$ 
Table 2 Top 20 Annotated Transcripts Downregulated in Light-induced Plasmodia

\begin{tabular}{|c|c|c|c|c|c|c|}
\hline Contig ID & SwissProt & Annotation & hits(D) & hits(L) & relD/relt & P-value \\
\hline contig10338_1 & P36618 & Cell division control protein 16, CDC16 & 40 & 1 & 31.7301 & 7.48E-10 \\
\hline contig10470_1 & P20072 & Annexin A7 & 68 & 2 & 26.9706 & $1.54 \mathrm{E}-15$ \\
\hline contig00397_1 & Q5BMR2 & Phospholipase D (PLD1) & 62 & 2 & 24.5908 & 4.31E-14 \\
\hline contig00525_1 & Q7EYV7 & Poly ADP-ribose polymerase 1 (PARP-1) & 244 & 10 & 19.3554 & $5.19 \mathrm{E}-49$ \\
\hline contig11321_1 & P38750 & Uncharacterized transporter YHLO08C & 24 & 1 & 19.0381 & 5.43E-06 \\
\hline contig00901_1 & P16064 & Subtilisin inhibitor 1, ASI-I & 21 & 1 & 16.6583 & 2.79E-05 \\
\hline PpolyN1a03a12 & Q07346 & Glutamate decarboxylase, GAD & 20 & 1 & 15.8651 & 4.80E-05 \\
\hline PpolyN1a02c07 & P34121 & Coactosin, coaA & 56 & 3 & 14.8074 & $1.07 \mathrm{E}-11$ \\
\hline contig11574_1 & P39749 & Flap endonuclease 1, FEN-1 & 18 & 1 & 14.2786 & 0.000141 \\
\hline contig10414_1 & Q5UNX2 & Putative ankyrin repeat protein, YL715 & 90 & 5 & 14.2786 & 8.82E-18 \\
\hline contig03548_1 & O49286 & F-box/LRR-repeat protein 5, FBL5 & 17 & 1 & 13.4853 & 0.000242 \\
\hline contig00369_1 & Q80U58 & Pumilio homolog 2 & 17 & 1 & 13.4853 & 0.000242 \\
\hline contig10457_1 & Q8WN03 & Kv channel-interacting protein 2, Kcnip2 & 16 & 1 & 12.6921 & 0.000412 \\
\hline contig00264_1 & P13466 & Actin-binding protein $120, \mathrm{ABP}-120$ & 32 & 2 & 12.6921 & $5.26 \mathrm{E}-07$ \\
\hline contig02333_1 & Q8RWN7 & POLTERGEIST Protein phosphatase $2 \mathrm{C} 32$, PP2C & 15 & 1 & 11.8988 & 0.000701 \\
\hline contig01650_1 & $\mathrm{O} 24496$ & Glyoxalase II, Glx II & 15 & 1 & 11.8988 & 0.000701 \\
\hline contig01322_1 & Q94B74 & NADH pyrophosphatase & 15 & 1 & 11.8988 & 0.000701 \\
\hline contig08310_1 & Q10MW3 & Pyruvate decarboxylase isozyme 2, PDC2 & 73 & 5 & 11.5815 & $6.84 \mathrm{E}-14$ \\
\hline contig00558_1 & P18281 & Actobindin & 29 & 2 & 11.5022 & $2.55 \mathrm{E}-06$ \\
\hline contig11873_1 & 010296 & Apoptosis inhibitor 1, IAP-1 & 28 & 2 & 11.1055 & 4.30E-06 \\
\hline
\end{tabular}

Transcripts with unambiguous annotations, significant differential expression $(\mathrm{P}<0.05)$, and that possess the highest levels of downregulation (relD/relL $>1.0)$, are listed. BLAST2GO [15] automatic annotations were used, and manual corrections of annotations were included in some cases.

Table 3 Top 20 Annotated Transcripts Upregulated in Light-induced Plasmodia

\begin{tabular}{|c|c|c|c|c|c|c|}
\hline Contig ID & SwissProt & Annotation & hits(D) & hits(L) & relL/relD & P-value \\
\hline PpolyN1d39e07 & O08623 & Sequestosome-1, SQSTM1 & 3 & 171 & 71.6971 & $2.03 \mathrm{E}-56$ \\
\hline contig02685_1 & Q54IV3 & ATP-dependent RNA helicase, DDX42 & 1 & 37 & 46.8103 & $7.87 \mathrm{E}-13$ \\
\hline PpolyN1d106 h10 & Q9U1K1 & Spire & 23 & 813 & 44.5504 & $5.40 \mathrm{E}-250$ \\
\hline PpolyN1a08 g07 & O08849 & Regulator of G-protein signaling 2, RGS2 & 1 & 22 & 27.8276 & 9.99E-08 \\
\hline contig05590_1 & Q8H100 & ADP-ribosylation factor GTPase-activating, AGD8 & 1 & 21 & 26.5690 & $2.17 \mathrm{E}-07$ \\
\hline PpolyN1a14d12 & Q07283 & Trichohyalin, TRHY & 1 & 20 & 25.2931 & 4.69E-07 \\
\hline contig11781_1 & Q55D99 & Serine/threonine-protein kinase, pakA & 2 & 34 & 21.5086 & $8.84 \mathrm{E}-11$ \\
\hline contig06420_1 & Q9UUG5 & Myosin regulatory light chain 1, MLR1 & 1 & 17 & 21.5000 & 4.69E-06 \\
\hline contig08470_1 & Q54MI7 & Uncharacterized protein DDB_G0285917 & 1 & 16 & 20.2414 & $1.01 \mathrm{E}-05$ \\
\hline contig12553_1 & Q5R826 & Transmembrane protein 63A, TM63A & 20 & 308 & 19.4149 & $1.89 \mathrm{E}-83$ \\
\hline PpolyN1d18d06 & Q05924 & Dosage-dependent cell cycle regulator 2, DCR2 & 1 & 15 & 18.9828 & $2.15 \mathrm{E}-05$ \\
\hline contig08799_1 & Q43207 & Rotamase, FKBP70 & 1 & 14 & 17.7069 & $4.59 \mathrm{E}-05$ \\
\hline contig12445_1 & Q7S045 & Non-histone chromosomal protein 6, NHP6 & 1 & 13 & 16.4483 & $9.76 \mathrm{E}-05$ \\
\hline$\overline{\text { contig11110_1 }}$ & P54678 & Calcium-transporting ATPase, PAT1 & 1 & 13 & 16.4483 & $9.76 \mathrm{E}-05$ \\
\hline contig08929_1 & Q39572 & Ras-related YPTC6 & 1 & 13 & 16.4483 & $9.76 \mathrm{E}-05$ \\
\hline contig08360_1 & Q6TQE1 & Zinc finger- containing protein $18, \mathrm{NHN} 1$ & 1 & 12 & 15.1724 & $2.06 \mathrm{E}-04$ \\
\hline contig04102_1 & Q9D0C1 & Rab RING finger 7, RR7 & 4 & 47 & 14.8026 & $2.86 \mathrm{E}-13$ \\
\hline contig03233_1 & P06704 & Cell division control protein 31, CDC31 & 2 & 23 & 14.5517 & $3.44 \mathrm{E}-07$ \\
\hline contig02500_1 & Q5UPW6 & Putative FNIP repeat-containing protein, L281 & 2 & 23 & 14.5517 & $3.44 \mathrm{E}-07$ \\
\hline contig08917_1 & Q9PTW9 & Proteasome subunit alpha type-7, PSMA7 & 1 & 11 & 13.9138 & $4.35 \mathrm{E}-04$ \\
\hline
\end{tabular}

A list of transcripts with unambiguous annotations, significant differential expression $(P<0.05)$ with the highest levels of upregulation (relL/relD $>1.0)$, is shown. Annotations, SwissProt accessions, hit counts, and probability values follow the same convention as in Table 2 . 


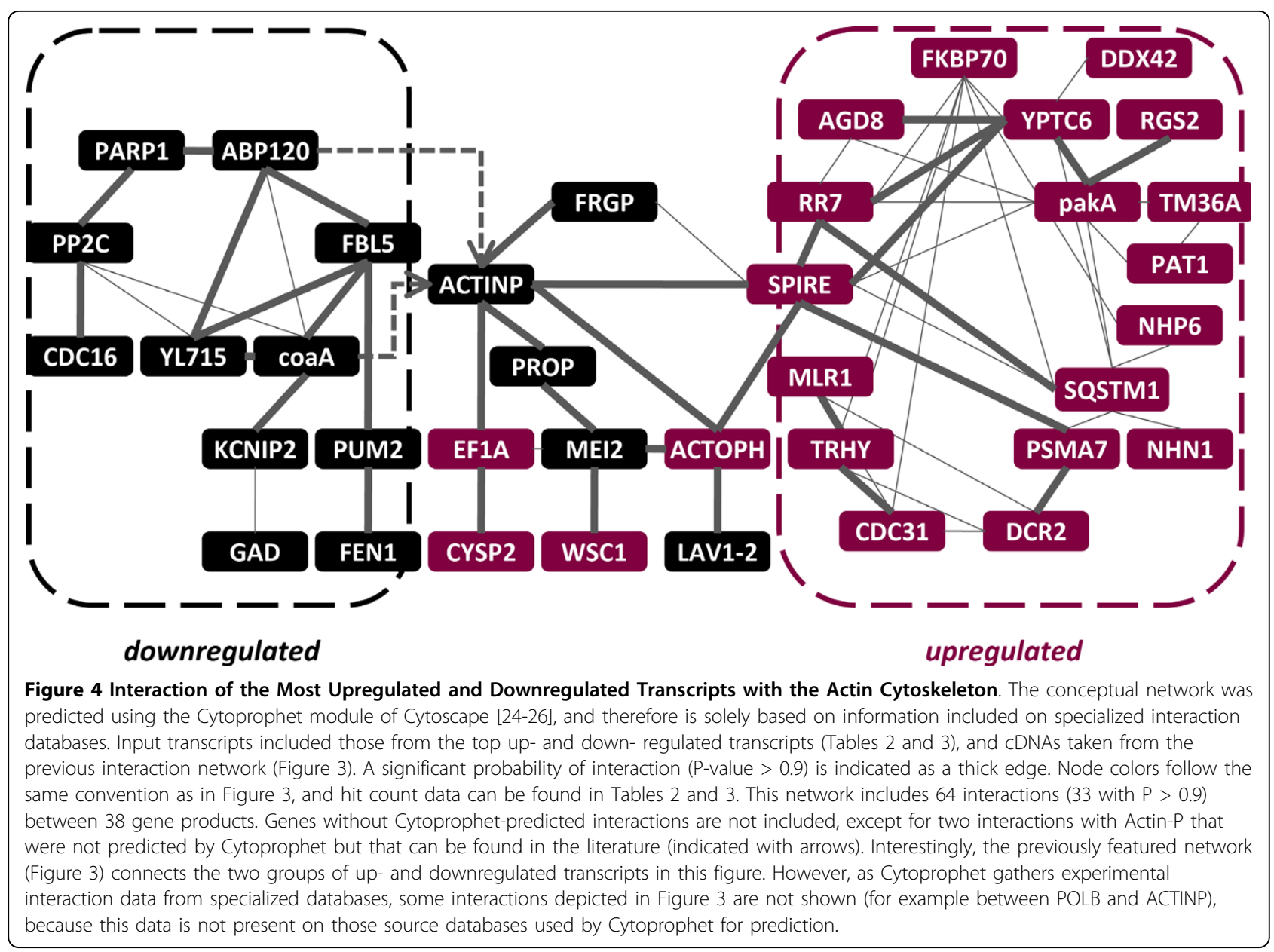

that leads to the activation of a plasmodium-specific transglutaminase, which separates damaged areas of a plasmodium [50]. Other transcripts encoding orthologs of calcium-binding proteins, such as KCNIP2 and GAD, were also downregulated in the photoinduced plasmodium and have not been previously described for in Physarum. KCNIP2 encodes a potassium channelinteracting protein that probably modulates channels density in a $\mathrm{Ca}^{2+}$ - dependent manner. In turn, the activation of glutamate decarboxylase (GAD) by calciumbound calmodulin (CaM) is required for normal growth in plants [51]. Previous studies have shown that the intracellular increase of calcium levels is correlated with increased concentrations of cAMP and with sporulation and differentiation in both Physarum and Dictyostelium [52,53]. Moreover, actin filament crosslinking is affected by changes in intracellular calcium levels, which ultimately influences the cell contractility [54]. Therefore it seems possible that these calcium-binding proteins coordinate the $\mathrm{Ca}^{2+}$ release as a means to influence the cell contractility through the interaction with the actin cytoskeleton (Figure 4).
Furthermore, the upregulated subset of calcium-binding proteins included MLR1, which inhibits cytokinesis in yeasts; trychohyalin (TRHY), which is involved in its own calcium-dependent processing during differentiation; and the Dictyostelium PAT1 ATPase. PAT1 is localized in the membrane of contractile vacuoles, and is a component of a calcium sequestration and excretion pathway, which functions to help maintain homeostasis, especially under conditions of $\mathrm{Ca}^{2+}$ stress [55]. Thus these are candidates to control the intracellular calcium levels after light induction of starved plasmodia.

\section{Actin-binding proteins associated to development are overexpressed in the light-induced plasmodium} After photoinduction, a group of actin-binding proteins is upregulated including the elongation factor $1 \alpha$ (EF1A), Spire, and actophorin (Figures 3 and 4; Table 3; [Additional File 5: Table S1]). Spire is a Drosophila gene involved in development through actin assembly. This gene is also widely distributed across the metazoan genomes. Spire mammalian isoforms are MAP kinase substrates, and data suggest that Spire evolved as an 
alternative independent mechanism of actin polymerization, necessary for cell polarization in multicellular organisms [56]. Actophorin, in turn, binds actin monomers and separates actin filaments in a dose-dependent manner. Phosphorylation of actophorin blocks actin binding [57]. In turn, EF1A, aside from its role in the protein synthesis, has a separate conserved actin-binding activity in eukaryota, initially observed in Dictyostelium [58], where it is predominantly found in actin-bound form [59]. EF1A regulates the stoichiometry of cytoskeletal components, and the conservation of the EF1A-actin interaction across eukaryotes suggests its importance for cytoskeletal maintenance [60]. Overexpression of EF1A in yeast results in effects on cell growth, and influences the actin distribution, morphology and budding in a dosage-dependent manner, although this increase of EF1A has no effect over the protein synthesis [61]. In addition, changes in cytoskeletal redistribution of EF1A seem to be linked to the differentiation status, where the association between EF1A and microtubules gradually increases in differentiating cultures [62]. Furthermore, EF1A stimulates actin remodeling and induces the formation of filopodia [63], and possibly connects these processes with signaling pathways [64].

We noticed that two coexpressed transcripts (the cysteine proteinase CYSP2 and the developmentally regulated gene $\mathrm{CudA}$ ) are related to EF1A. First, cysteine proteinases are believed to participate in protein cleavage during the differentiation of Dictyostelium as a response to starvation [65], and these peptidases were copurified with EF1A in yeasts [66]. CudA, on the other hand, is associated to the transition from slug migration to culmination in Dictyostelium. CudA expression levels depend on local cAMP concentration [67]. Recent evidences show that CudA contains a novel DNA-binding site that is distantly related to the metazoan STAT domains, which participate in the regulation of developmentally controlled genes [68], and whose orthologs coexpress with EF1A [69]. Yamada et al. [68] also proved a relationship between Dictyostelium CudA and a cDNA from Physarum, which corresponds to the contig reported here as a CudA ortholog. For these reasons, EF1A could work as a link between regulation of the protein synthesis, cytoskeletal maintenance, and signal transduction in slime molds (Figure 3).

Other developmentally regulated transcripts associated to the actin cytoskeleton included the cell wall integrity and stress response component (WSC1), which is a yeast membrane protein that acts as a sensor of cell wall damage [70], and CDC31, a constituent of the nuclear pore complex that is also involved in the maintenance of cell morphology (Table 3 and Figure 4). WSC1 is essential to keep the cell integrity, behaving like a stress-specific signal transducer that is involved in the reorganization of the actin cytoskeleton in response to osmotic shock [71,72]. WSC1 is involved in the depolarization of the actin cytoskeleton [72], and, like CDC16 (downregulated in light-induced plasmodia), is entailed in cytokinesis [73].

\section{GTP signaling genes involved in different processes are upregulated in the light-induced plasmodium}

Orthologs of certain genes highly upregulated in lightinduced plasmodia are involved in signal transduction. These include transcripts linked to the GTP signaling (AGD8, YPTC6, RGS2), kinases (pakA) and phosphatases (DCR2). The serine/threonine-kinase pakA is a regulator of the myosin component of the cytoskeleton, required for cytokinesis and the regulation of the cytoskeleton during chemotaxis in Dictyostelium [74]. In turn, the yeast dosage-dependent cell cycle regulator 2 (DCR2), is a phosphatase whose increased dosage alters cell cycle progression, while its loss delays the progression in the G1 phase [75]. In addition, upregulated GTP signaling transducers included a putative GTPase- activating protein from Arabidopsis (AGD8); a Chlamydomonas GTPbinding protein (YPTC6); and RGS2, which acts as a negative regulator of G-protein signaling, a function that is evolutionarily conserved in yeast, C. elegans and mammals. Increased RGS2 expression is primarily mediated by the cAMP/PKA pathway [76], therefore it is possible that RGS2 is carrying out similar tasks in slime molds, where it could work in coordination with the other transducers, as hypothesized in Figure 4.

\section{Transcripts annotated for cell death are overrepresented in the light-induced plasmodium}

Comparison of GO terms between up- and downregulated groups showed that transcripts annotated for 'cell development' (GO:0048468), 'cell death' (GO:0008219) and 'death' (GO:0016265) were overrepresented in the upregulated group [Additional File 6: Table S2]. However, all these ontologies belong to the same hierarchy, meaning that 'cell death' can be the product of either development or organismal death, and hence 'cell death' is the only difference between both expression groups. One of these cDNAs annotated for 'cell death' is Sequestosome 1, which is also included on the list of upregulated transcripts (Table 3 ). Sequestosome 1, also known as p62, is a multifunctional protein that targets polyubiquitinated proteins to degradation by proteasomes and autophagy [77]. p62 knockouts significantly increased cell death [78], and this is probably linked to the interaction with atypical protein kinase $\mathrm{C}$ isoforms that are involved in pathways that control differentiation and apoptosis [79]. Therefore it is likely that this gene product regulates cell death pathways linked to the commitment for sporulation. 
Furthermore, other highly upregulated genes are also functionally linked to the protein turnover. These include the FKBP70 rotamase, which accelerates the folding of proteins during synthesis; the PSMA7 proteasome subunit, which together with the other subunits, suffer changes during the meiotic cell cycle [80]; and the endosome-lysosome vesicle traffic-related RR7 [81]. It is likely then that these gene products, together with Sequestosome 1, are linked to the control of differentiation through post-transcriptional regulation.

\section{Conclusions}

The gain of sporulation-competence of Physarum plasmodia involves growth arrest, condensation of constituents, and mitosis and is a prerequisite before sporulation can be induced by light [27]. Physarum gene expression has been shown to be cell type-specific, but existing studies have been focused only on individual genes [2-4]. Previously, we reported a library of 5,856 sequences obtained from plasmodia competent for the induction of sporulation [6]. In the present study we used the massive parallel sequencing technology at the level of the whole transcriptome $[7,8]$ in order to identify global changes in expression that occur during light-induced sporulation of Physarum. We integrated the differentially expressed cDNAs into networks using interaction information from orthologs and the literature. We found that after light induction of a plasmodium the expression of transcripts linked to cell division and DNA repair is downregulated. In contrast, lightinduction stimulated the expression of genes associated with protein turnover (proteases and proteasome transcripts), genes related to cell cycle progression, and genes involved in the maintenance of cell integrity and cytokinesis. These latter gene products might protect the cell against osmotic shock. Additionally, different groups of calcium-binding proteins are either down- or upregulated after light exposure. These gene products are candidates to control the intracellular calcium levels during sporulation. We postulate that these changes are associated with a network of actin-binding proteins (Figures 3 and 4), the components of which are differentially regulated upon plasmodial photoinduction. It seems that these gene products accomplish different tasks in each stage: the reorganization of the subcellular compartments in order to inhibit migration during starvation on one hand, and cell polarization and cytoskeletal redistribution after photoinduction mediated by a group of actin-binding proteins on the other. We expect that the precise representation of the proposed interaction networks may become available as gene knockout experiments, proteomic data, and comparative interactomics are integrated in future studies of this organism.

\section{Methods}

Culture and light-induction of plasmodial cells

Physarum plasmodia of the white strain (LU897 $\times$ LU898) were hatched from spherules, and grown as microplasmodial suspensions for four days. The plasmodial mass was then applied to starvation agar plates. Microplasmodia spontaneously fused to give a single plasmodium on each plate. Plasmodia were then starved for six days in the dark at $22^{\circ} \mathrm{C}$ to obtain maximal competence for sporulation. To verify the sporulation-competent state, plasmodia were cut into two halves. One half was immediately frozen in liquid nitrogen for RNA extraction, and the other half was returned to the dark and incubated until the next day to verify that the plasmodium had not been induced to sporulation. To obtain light-induced plasmodia, competent plasmodia were irradiated for $30 \mathrm{~min}$ with far red light and then returned to the dark. Six hours after the start of irradiation, plasmodia were cut into two halves. One half was frozen in liquid nitrogen for RNA extraction. The other half was returned to the dark and incubated until the next day to verify the sporulation status $[3,4]$.

\section{cDNA Library Construction and Sequencing}

Transcript poly $(\mathrm{A})^{+}$RNAs were isolated by oligo-dT chromatography. cDNAs were prepared from these RNAs by the full-length enriched synthesis method (vertis Biotechnologie, Freising- Weihenstephan, Germany). First strand cDNA was synthesized using oligo(dT) adapter primers and MMLV H-reverse transcriptase. Following RNA hydrolysis, an adapter primer was annealed to the 3 ' end, and the produced fragments were PCR-amplified for 22 cycles with a proofreading enzyme. The cDNA libraries were then directly sequenced using the 454 GS FLX system (Roche Diagnostics, Mannheim, Germany) [7]. Chromatograms were scored for quality, and the produced sequences were trimmed of adapter sequences, and coassembled into contigs using previously available transcriptomic data [6]. For expression comparisons we obtained for each contig: $(i)$ the number of reads (which we define as "hit counts") in both libraries; (ii), their relative frequencies (reads of a given contig divided by the total number of reads); and (iii) their relative frequencies. Statistical significance between the two hit counts for each contig species was then assessed [9].

\section{Sequence Annotation and Network Inference}

Similarity searches against protein databases were performed using BLASTX implemented in the MIGenAS tool [82] (e-value $1 \times 10-3)$. We utilized nine protein databases in this comparison: Swiss-Prot and TrEMBL (versions 56.3 and 39.3) [12], dictyBase [14] and RefSeq database subsets: mammalian, other vertebrates, 
invertebrate, protozoa, plant and microbial (release 31) [13]. Functional annotation was carried out using BLAST2GO (version 2.2.3) [16]. This procedure consisted of a similarity search against the non-redundant GenBank database [10], using BLASTX (e-value $1 \times 10-3$ ), followed by Gene Ontology (GO) [15] mappings extracted from similarity results and InterPro domain matches (InterPro release 18.0) [17]. Annotation of sequences (cutoff value $1 \times 10-6$ ) was followed by their validation, and these annotations were extended using ANNEX [83]. Statistical analysis of GO annotations between differentially expressed cDNAs was carried out using the Fisher exact test, as implemented in the GOSSIP module [18] of BLAST2GO. Sequences were also categorized in metabolic and signaling pathways, via similarity search against orthologs present in the KEGG database [19] using the KAAS server [20]. In this case, we employed default parameters for ESTs. KEGG orthologs (KOs) were then plotted into the whole metabolic atlas, utilizing the KEGG mapping tool [21]. Putative networks of correlated genetic interactions were generated from annotation information, using the MLE algorithm [26], as implemented in the Cytoprophet plugin [24] of Cytoscape [25].

Additional file 1: Figure S1. Overview of the Experimental Design. A summary of experiments and computational analyses is depicted. RNA samples were taken from competent plasmodia after six days of starvation in the dark, and from competent plasmodia at six hours after exposition to a 30 minutes pulse of red light ( $\geq 700 \mathrm{~nm}$ ) (1) [3]. cDNAs were synthesized from extracted RNAs (2), and sequenced and quantitated using the 454 Life Sciences platform (3) [78]. Contigs generated were then annotated at every bioinformatic level (4), and network interactions (5) were obtained both by a combination of manual curation of literature, expression data, and predictions from annotations (Powerpoint file).

Click here for file

[http://www.biomedcentral.com/content/supplementary/1471-2164-11115-S1.ppt ]

Additional file 2: Transcript Abundance Data. Hit counts obtained from pyrosequencing, including statistical analyses (Excel spreadsheet). Click here for file

[http://www.biomedcentral.com/content/supplementary/1471-2164-11115-S2.xIs ]

Additional file 3: Figure S2. Hits Distribution of Transcript Species. The distribution of pyrosequencing hit counts respect to the number of transcript species on each library (starvation and light-induced) is depicted on a semi-logarithmic scale. Hit counts are included in the adjacent upper ranges to the right; for example, transcripts with 2 hits are present in the 2-5 range. Similar distributions of contig species were found on both libraries, and most transcripts were represented by 1 to 5 hits only (Powerpoint file).

Click here for file

[http://www.biomedcentral.com/content/supplementary/1471-2164-11115-S3.ppt ]

Additional file 4: Similarity Results. Results of the similarity analyses against protein databases from sequences with hit counts data (Excel spreadsheet).

Click here for file

[http://www.biomedcentral.com/content/supplementary/1471-2164-11115-S4.Xls ]
Additional file 5: Table S1. Annotated transcripts with relative frequencies higher than $\mathbf{0 . 0 0 5}$. A list of transcripts obtained from the scatterplot of relative frequencies (Figure 1) is depicted. Annotations, hit counts, and probability values follow the same convention as in Table 2 (Word document).

Click here for file

[http://www.biomedcentral.com/content/supplementary/1471-2164-11115-S5.doc ]

Additional file 6: Table S2. Overrepresented Gene Ontology terms in Upregulated Transcripts. Full lists of GO terms from up- and downregulated contigs were compared against each other using the Fisher's exact test from the GOSSIP program [18], as implemented in BLAST2GO [16]. A two-tailed test with the false discovery rate (FDR) filter was employed. The number of GO-annotated transcripts used for comparison between up- (Test) and downregulated (Ref) groups of cDNAs is shown. All overrepresented GO terms belong to the biological process (BP) category (Word document).

Click here for file

[http://www.biomedcentral.com/content/supplementary/1471-2164-11115-S6.doc ]

Additional file 7: Transcriptome Annotation. Annotations of all sequences, including those with or without hit counts data (Excel spreadsheet)

Click here for file

[http://www.biomedcentral.com/content/supplementary/1471-2164-11115-S7.xls ]

Additional file 8: Table S3. Top 20 Transcripts Downregulated in Light-induced Plasmodia. Transcripts with the highest rates of downregulation (relD/relL > 1.0), are listed. BLAST2GO [15] automatic annotations were used, and manual corrections were included in some cases. Transcripts with unknown orthologs are described with "-NA-." Annotations, SwissProt accessions, hit counts, and probability values follow the same convention as in Table 2 (Word document).

Click here for file

[http://www.biomedcentral.com/content/supplementary/1471-2164-11115-S8.doc ]

Additional file 9: Table S4. Top 20 Transcripts Upregulated in Lightinduced Plasmodia. Transcripts with the highest rates of upregulation (relL/relD > 1.0), are listed. BLAST2GO [15] automatic annotations were used, and manual corrections were included in some cases. Columns follow the same convention as in Table S3 (Word document).

Click here for file

[http://www.biomedcentral.com/content/supplementary/1471-2164-11115-S9.doc ]

\section{Abbreviations}

EF1A: elongation factor 1- alpha; WSC1: cell wall integrity and stress response component; mei2: yeast meiosis- related protein; PKA: CAMPdependent protein kinase A; STAT: Signal transducer and activator of transcription; GO: Gene Ontology; FDR: false discovery rate; KEGG: Kyoto Encyclopedia of Genes and Genomes; KAAS: KEGG Automatic Annotation Server; KO: KEGG ortholog.

\section{Acknowledgements}

We thank Markus Rampp (Rechenzentrum Garching of the Max Planck Society), for his support during the computational analysis. WIB is an International Max Planck Research School - Otto von Guericke University fellow. The experimental part of the study was supported by a cooperation contract with the Max Planck Society.

\section{Author details}

${ }^{1}$ International Max Planck Research School, Magdeburg, Germany. ${ }^{2}$ Leibniz Institute for Age Research, Fritz Lipmann Institute, Jena, Germany. ${ }^{3}$ Institute for Biochemistry University of Cologne, Joseph-Stelzmann-Str. 52, Cologne, Germany. ${ }^{4}$ Max Planck Institute for Dynamics of Complex Technical Systems and Magdeburg Centre for Systems Biology (MaCS), Otto von Guericke University, Magdeburg, Germany. ${ }^{5}$ Berlin Center for Genomics in Biodiversity 
Research Leibniz Institute for Freshwater Ecology and Inland Fisheries, Müggelseedamm 301, D-12587 Berlin, Germany. ${ }^{6}$ Magdeburg Centre for Systems Biology (MaCS), Otto von Guericke University, Sandtorstr. 1, D-39106 Magdeburg, Germany.

\section{Authors' contributions}

WM conceived of the research. GG and WM designed the study, and IB contributed to the experimental design. WM cultured the cells, isolated RNA, and coordinated the project. GG performed the sequencings, contig assemblies and quantitations. SM and IB carried out statistical analyses and performed similarity searches. IB annotated the transcriptome, as well as modeled interaction networks. IB wrote the paper, and WM and GG helped with the manuscript preparation. All authors read and approved the final manuscript.

\section{Authors' Information}

Website for Affiliation 5: http://www.ovgu.de/ag-marwan/

Received: 30 October 2009

Accepted: 17 February 2010 Published: 17 February 2010

\section{References}

1. Burland TG, Solnica-Krezel L, Bailey J, Cunningham DB, Dove WF: Patterns of inheritance development and the mitotic cycle in the protist Physarum polycephalum. Adv Microbial Physiol 1993, 35:1-69.

2. Martel R, Tessier A, Pallotta D, Lemieux G: Selective gene expression during sporulation of Physarum polycephalum. J Bacteriol 1988, 170:4784-4790.

3. Kroneder R, Cashmore AR, Marwan W: Phytochrome-induced expression of lig1 a homologue of the fission yeast cell-cycle checkpoint gene hus1 is associated with the developmental switch in Physarum polycephalum plasmodia. Curr Genet 1999, 36:86-93.

4. Golderer G, Werner ER, Leitner S, Grobner P, Werner-Felmayer G: Nitric oxide synthase is induced in sporulation of Physarum polycephalum. Genes Dev 2001, 15:1299-1309.

5. Watkins RF, Gray MW: Sampling gene diversity across the supergroup amoebozoa: Large EST data sets from Acanthamoeba castellanii, Hartmannella vermiformis, Physarum polycephalum, Hyperamoeba dachnaya and Hyperamoeba sp. Protist 2008, 159:269-281.

6. Glockner G, Golderer G, Werner-Felmayer G, Meyer S, Marwan W: A first glimpse at the transcriptome of Physarum polycephalum. BMC Genomics 2008, 9:6.

7. Margulies M, Egholm M, Altman WE, Attiya S, Bader JS, Bemben LA, Berka J, Braverman MS, Chen YJ, Chen Z, Dewell SB, Du L, Fierro JM, Gomes XV, Godwin BC, He W, Helgesen S, Ho CH, Irzyk GP, Jando SC, Alenquer MLI, Jarvie TP, Jirage KB, Kim JB, Knight JR, Lanza JR, Leamon JH, Lefkowitz SM, Lei $\mathrm{M}, \mathrm{Li}$ J, et al: Genome sequencing in microfabricated high-density picolitre reactors. Nature 2005, 437:376-80.

8. Wang Z, Gerstein M, Snyder M: RNA-Seq: a revolutionary tool for transcriptomics. Nature Rev Genet 2009, 10:57-63.

9. Audic S, Claverie JM: The significance of digital gene expression profiles. Genome Res 1997, 7(10):986-95.

10. Benson DA, Karsch-Mizrachi I, Lipman DJ, Ostell J, Wheeler DL: GenBank. Nucleic Acids Res 2008, 36:D25-30.

11. Altschul SF, Madden TL, Schaffer AA, Zhang J, Zhang Z, Miller W, Lipman DJ: Gapped Blast and Psi-Blast: a new generation of protein database search programs. Nucleic Acids Res 1997, 25:3389-3402.

12. Boeckmann B, Bairoch A, Apweiler R, Blatter MC, Estreicher A, Gasteiger E, Martin MJ, Michoud K, O'Donovan C, Phan I, Pilbout S, Schneider M: The Swiss-Prot protein knowledgebase and its supplement TrEMBL in 2003. Nucleic Acids Res 2003, 31:365-370.

13. Pruitt KD, Tatusova T, Maglott DR: NCBI reference sequences (RefSeq): $A$ curated non-redundant sequence database of genomes transcripts and proteins. Nucleic Acids Res 2007, 35:D61-5.

14. Chisholm RL, Gaudet P, Just EM, Pilcher KE, Fey P, Merchant SN, Kibbe WA dictybase, the model organism database for Dictyostelium discoideum. Nucleic Acids Res 2006, 34:D423-7.

15. Ashburner M, Ball CA, Blake JA, Botstein D, Butler H, Cherry JM, Davis AP, Dolinski K, Dwight SS, Eppig JT, Harris MA, Hill DP, Issel-Tarver L, Kasarskis A, Lewis S, Matese JC, Richardson JE, Ringwald M, Rubin GM, Sherlock G: Gene ontology: A tool for the unification of biology. Nature Genet 2000, 25:25-29.

16. Gotz S, Garcia-Gomez JM, Terol J, Williams TD, Nagaraj SH, Nueda MJ, Robles M, Talon M, Dopazo J, Conesa A: High-throughput functional annotation and data mining with the Blast2GO suite. Nucleic Acids Res 2008, 36(10):3420-35.

17. Hunter S, Apweiler R, Attwood TK, Bairoch A, Bateman A, Binns D, Bork P, Das U, Daugherty L, Duquenne L, Finn RD, Gough J, Haft D, Hulo N, Kahn D, Kelly E, Laugraud A, Letunic I, Lonsdale D, Lopez R, Madera M, Maslen J, McAnulla C, McDowall J, Mistry J, Mitchell A, Mulder N, Natale D, Orengo $C$, Quinn $A F$, et al: Interpro: the integrative protein signature database. Nucleic Acids Res 2009, 37:D211-5.

18. Bluthgen N, Brand K, Cajavec B, Swat M, Herzel H, Beule D: Biological profiling of gene groups utilizing gene ontology. Genome Informat 2005, 16:106-115.

19. Kanehisa M, Araki M, Goto S, Hattori M, Hirakawa M, Itoh M, Katayama T, Kawashima S, Okuda S, Tokimatsu T, Yamanishi Y: KEGG for linking genomes to life and the environment. Nucleic Acids Res 2008, 36:D480-4.

20. Moriya Y, Itoh M, Okuda S, Yoshizawa AC, Kanehisa M: KAAS: an automatic genome annotation and pathway reconstruction server. Nucleic Acids Res 2007, 35:W182-5.

21. Okuda S, Yamada T, Hamajima M, Itoh M, Katayama T, Bork P, Goto S, Kanehisa M: KEGG atlas mapping for global analysis of metabolic pathways. Nucleic Acids Res 2008, 36:W423-6.

22. Ge H, Liu Z, Church GM, Vidal M: Correlation between transcriptome and interactome mapping data from Saccharomyces cerevisiae. Nature Genet 2001, 29:482-486.

23. Fraser $H B$, Hirsh AE, Wall DP, Eisen MB: Coevolution of gene expression among interacting proteins. Proc Natl Acad Sci USA 2004, 101:9033-9038

24. Morcos F, Lamanna C, Sikora M, Izaguirre J: Cytoprophet: a Cytoscape plug-in for protein and domain interaction networks inference. Bioinformatics 2008, 24(19):2265-6.

25. Shannon P, Markiel A, Ozier O, Baliga NS, Wang JT, Ramage D, Amin N, Schwikowski B, Ideker T: Cytoscape: a software environment for integrated models of biomolecular interaction networks. Genome Res 2003, 13(11):2498-504.

26. Deng M, Mehta S, Sun F, Chen T: Inferring domain-domain interactions from protein-protein interactions. Genome Res 2002, 12(10):1540-8.

27. Bailey J: Building a plasmodium: Development in the acellular slime mould Physarum polycephalum. Bio Essays 1997, 19:985-992.

28. Bailey J, Cook LJ, Kilmer-Barber R, Swanston E, Solnica-Krezel L, Lohman K, Dove WF, Dee J, Anderson RW: Identification of three genes expressed primarily during development in Physarum polycephalum. Arch Microbiol 1999, 172:364-376.

29. Shirai $Y$, Sasaki N, Kishi $Y$, Izumi A, Itoh $K$, Sameshima M, Kobayashi $T$, Murakami-Murofushi K: Regulation of levels of actin threonine phosphorylation during life cycle of Physarum polycephalum. Cell Motil Cytoskel 2006, 63:77-87.

30. Binette F, Benard M, Laroche A, Pierron G, Lemieux G, Pallotta D: Cellspecific expression of a profilin gene family. DNA Cell Biol 1990, 9:323-334.

31. de Hostos EL, Bradtke B, Lottspeich F, Gerisch G: Coactosin, a 17 kda factin binding protein from Dictyostelium discoideum. Cell Motil Cytoskel 1993, 26:181-191.

32. Vandekerckhove J, Van Damme J, Vancompernolle K, Bubb MR, Lambooy PK, Korn ED: The covalent structure of Acanthamoeba actobindin. J Biol Chem 1990, 265:12801-12805.

33. Rohrig U, Gerisch G, Morozova L, Schleicher M, Wegner A: Coactosin interferes with the capping of actin filaments. FEBS Lett 1995, 374:284-286.

34. Brulle F, Cocquerelle C, Mitta G, Castric V, Douay F, Lepretre A, Vandenbulcke F: Identification and expression profile of gene transcripts differentially expressed during metallic exposure in Eisenia fetida coelomocytes. Dev Comp Immunol 2008, 32:1441-1453.

35. Khaire $N$, Muller R, Blau-Wasser R, Eichinger L, Schleicher M, Rief M, Holak TA, Noegel AA: Filamin-regulated f-actin assembly is essential for morphogenesis and controls phototaxis in Dictyostelium. I Biol Chem 2007, 282:1948-1955.

36. T'Jampens D, Bailey J, Cook LJ, Constantin B, Vandekerckhove J, Gettemans J: Physarum amoebae express a distinct fragmin-like actin- 
binding protein that controls in vitro phosphorylation of actin by the actin- fragmin kinase. Eur J Biochem 1999, 265:240-250.

37. Howe A: Regulation of actin-based cell migration by cAMP/PKA. Biochim Biophys Acta 2004, 1692(2-3):159-174.

38. Aubry L, Firtel R: Integration of signaling networks that regulate Dictyostelium differentiation. Ann Rev Cell Dev Biol 1999, 15:469-517.

39. Stettler S, Warbrick E, Prochnik S, Mackie S, Fantes P: The wis1 signal transduction pathway is required for expression of cAMP-repressed genes in fission yeast. J Cell Sci 1996, 109:1927-1935.

40. Fujioka $H$, Shimoda C: A mating-type-specific sterility gene map 1 is required for transcription of a mating-type gene mat1-Pi in the fission yeast Schizosaccharomyces pombe. FEMS Microbiol Lett 1989, 51(1):45-8.

41. Christmann M, Tomicic MT, Origer J, Kaina B: Fen1 is induced p53 dependently and involved in the recovery from uv-light-induced replication inhibition. Oncogene 2005, 24:8304-8313.

42. Larsen E, Kleppa L, Meza TJ, Meza-Zepeda LA, Rada C, Castellanos CG, Lien GF, Nesse GJ, Neuberger MS, Laerdahl JK, William Doughty R, Klungland A: Early-onset lymphoma and extensive embryonic apoptosis in two domain-specific FEN1 mice mutants. Cancer Res 2008, 68:4571-4579.

43. Cerutti $L$, Simanis V: Asymmetry of the spindle pole bodies and spg $1 p$ gap segregation during mitosis in fission yeast. J Cell Sci 1999, 112:2313-2321.

44. Fankhauser C, Marks J, Reymond A, Simanis V: The S. pombe cdc16 gene is required both for maintenance of p34cdc2 kinase activity and regulation of septum formation: a link between mitosis and cytokinesis?. EMBO J 1993, 12:2697-2704.

45. Lin H, Spradling AC: A novel group of pumilio mutations affects the asymmetric division of germline stem cells in the Drosophila ovary. Development 1997, 124(12):2463-76

46. Jin J, Cardozo T, Lovering RC, Elledge SJ, Pagano M, Harper JW: Systematic analysis and nomenclature of mammalian F-box proteins. Genes Dev 2004, 18:2573-2580.

47. Meijer HJ, Latijnhouwers M, Ligterink W, Govers F: A transmembrane phospholipase D in Phytophthora; a novel PLD subfamily. Gene 2005, 350:173-182.

48. Yu LP, Miller AK, Clark SE: Poltergeist encodes a protein phosphatase $2 c$ that regulates clavata pathways controlling stem cell identity at Arabidopsis shoot and flower meristems. Curr Biol 2003, 13:179-188.

49. Iwasaki W, Sasaki H, Nakamura A, Kohama K, Tanokura M: Crystallization and preliminary $\mathrm{x}$-ray diffraction studies of a $40 \mathrm{kda}$ calcium binding protein specifically expressed in plasmodia of Physarum polycephalum. J Biochem 1999, 126:7-9.

50. Mottahedeh J, Marsh R: Characterization of 101-kda transglutaminase from Physarum polycephalum and identification of LAV1-2 as substrate. J Biol Chem 1998, 273:29888-29895.

51. Yap KL, Yuan T, Mal TK, Vogel HJ, Ikura M: Structural basis for simultaneous binding of two carboxy-terminal peptides of plant glutamate decarboxylase to calmodulin. J Mol Biol 2003, 328:193-204.

52. Schlatterer C, Gollnick F, Schmidt E, Meyer R, Knoll G: Challenge with high concentrations of cyclic AMP induces transient changes in the cytosolic free calcium concentration in Dictyostelium discoideum. J Cell Sci 1994, 107:2107-2115.

53. Renzel S, Esselborn S, Sauer HW, Hildebrandt A: Calcium and Malate Are Sporulation-Promoting Factors of Physarum polycephalum. J Bacteriol 2000, 182(24):6900-6905.

54. Furukawa R, Maselli A, Thomson SAM, Lim RWL, Stokes JV, Fechheimer M: Calcium regulation of actin crosslinking is important for function of the actin cytoskeleton in Dictyostelium. J Cell Sci 2003, 116:187-196.

55. Moniakis J, Coukell MB, Janiec A: Involvement of the Ca2+-ATPase PAT1 and the contractile vacuole in calcium regulation in Dictyostelium discoideum. J Cell Sci 1999, 112:405-414.

56. Quinlan ME, Heuser JE, Kerkhoff E, Mullins DD: Drosophila spire is an actin nucleation factor. Nature 2005, 433:382-388.

57. Blanchoin L, Robinson RC, Choe S, Pollard TD: Phosphorylation of Acanthamoeba actophorin (ADF/cofilin) blocks interaction with actin without a change in atomic structure. J Mol Biol 2000, 295(2):203-11.

58. Yang F, Demma M, Warren V, Dharmawardhane S, Condeelis J: Identification of an actin- binding protein from Dictyostelium as elongation factor 1a. Nature 1990, 347:494-496.
59. Edmonds BT, Bell A, Wyckoff J, Condeelis J, Leyh TS: The effect of F-actin on the binding and hydrolysis of guanine nucleotide by Dictyostelium elongation factor 1A. J Biol Chem 1998, 273(17):10288-95.

60. Gross SR, Kinzy TG: Improper organization of the actin cytoskeleton affects protein synthesis at initiation. Mol Cell Biol 2007, 27:1974-1989.

61. Munshi R, Kandl KA, Carr-Schmid A, Whitacre JL, Adams AE, Kinzy TG: Overexpression of translation elongation factor 1a affects the organization and function of the actin cytoskeleton in yeast. Genetics 2001, 157:1425-1436.

62. Bluem R, Schmidt E, Corvey C, Karas M, Schlicksupp A, Kirsch J, Kuhse J: Components of the translational machinery are associated with juvenile glycine receptors and are redistributed to the cytoskeleton upon aging and synaptic activity. J Biol Chem 2007, 282:37783-37793.

63. Jeganathan S, Morrow A, Amiri A, Lee JM: Eukaryotic elongation factor 1 a2 cooperates with phosphatidylinositol-4 kinase III beta to stimulate production of filopodia through increased phosphatidylinositol-45 bisphosphate generation. Mol Cell Biol 2008, 28:4549-4561.

64. Li P, Maines-Bandiera S, Kuo WL, Guan Y, Sun Y, Hills M, Huang G, Collins CC, Leung PC, Gray JW, Auersperg N: Multiple roles of the candidate oncogene ZNF217 in ovarian epithelial neoplastic progression. Int J Cancer 2007, 120(9):1863-73.

65. Datta S, Firtel RA: Identification of the sequences controlling cyclic AMP regulation and cell-type-specific expression of a prestalk-specific gene in Dictyostelium discoideum. Mol Cell Biol 1987, 7:149-159.

66. Pope SN, Lee IR: Yeast two-hybrid identification of prostatic proteins interacting with human sex hormone-binding globulin. J Steroid Biochem Mol Biol 2005, 94(1-3):203-8.

67. Fukuzawa M, Williams JG: Analysis of the promoter of the cudA gene reveals novel mechanisms of Dictyostelium cell type differentiation. Development 2000, 127:2705-2713.

68. Yamada Y, Wang HY, Fukuzawa M, Barton GJ, Williams JG: A new family of transcription factors. Development 2008, 135(18):3093-101.

69. Li P, Maines-Bandiera S, Kuo WL, Guan Y, Sun Y, Hills M, Huang G, Collins CC, Leung PC, Gray JW, Auersperg N: Multiple roles of the candidate oncogene ZNF217 in ovarian epithelial neoplastic progression. Int J Cancer 2007, 120:1863-1873.

70. Gualtieri T, Ragni E, Mizzi L, Fascio U, Popolo L: The cell wall sensor wsc1p is involved in reorganization of actin cytoskeleton in response to hypoosmotic shock in Saccharomyces cerevisiae. Yeast 2004, 21:1107-1120

71. Serrano R, Martin H, Casamayor A, Arino J: Signaling alkaline pH stress in the yeast Saccharomyces cerevisiae through the WSC1 cell surface sensor and the SLT2 MAPK pathway. J Biol Chem 2006, 281(52):39785-95.

72. Delley PA, Hall MN: Cell wall stress depolarizes cell growth via hyperactivation of RHO1. J Cell Biol 1999, 147(1):163-74.

73. Cerutti $L$, Simanis V: Asymmetry of the spindle pole bodies and spg1p gap segregation during mitosis in fission yeast. J Cell Sci 1999, 112:2313-2321.

74. Chung CY, Firtel RA: PakA, a putative pak family member, is required for cytokinesis and the regulation of the cytoskeleton in Dictyostelium discoideum cells during chemotaxis. J Cell Biol 1999, 147:559-576.

75. Pathak R, Bogomolnaya LM, Guo J, Polymenis M: Gid8p (dcr1p) and dcr2p function in a common pathway to promote start completion in Saccharomyces cerevisiae. Eukaryotic Cell 2004, 3:1627-1638.

76. Miles RR, Sluka JP, Santerre RF, Hale LV, Bloem L, Boguslawski G, Thirunavukkarasu K, Hock JM, Onyia JE: Dynamic regulation of RGS2 in bone: Potential new insights into parathyroid hormone signaling mechanisms. Endocrinology 2000, 141:28-36.

77. Seibenhener ML, Geetha T, Wooten MW: Sequestosome 1/p62 - More than just a scaffold. FEBS Lett 2007, 581(2):175-179.

78. Bjorkoy $G$, Lamark T, Brech A, Outzen $H$, Perander $M$, Overvatn $A$, Stenmark H, Johansen T: p62/SQSTM1 forms protein aggregates degraded by autophagy and has a protective effect on huntingtininduced cell death. J Cell Biol 2005, 171(4):603-14.

79. Puls A, Schmidt S, Grawe F, Stabel S: Interaction of Protein Kinase C zeta with ZIP, a novel Protein Kinase C- binding protein. Proc Natl Acad Sci USA 1997, 94:6191-6196.

80. Tokumoto M, Horiguchi R, Nagahama Y, Ishikawa K, Tokumoto T: Two proteins, a goldfish 20s proteasome subunit and the protein interacting with 26 s proteasome, change in the meiotic cell cycle. Eur J Biochem 2000, 267:97-103. 
81. Mizuno K, Kitamura A, Sasaki T: Rabring7, a novel rab7 target protein with a ring finger motif. Mol Biol Cell 2003, 14:3741-3752.

82. Rampp M, Soddemann T, Lederer H: The MIGenAS integrated bioinformatics toolkit for web-based sequence analysis. Nucleic Acids Res 2006, 34:W15-9.

83. Myhre $\mathrm{S}$, Tveit $\mathrm{H}$, Mollestad T, Laegreid A: Additional gene ontology structure for improved biological reasoning. Bioinformatics 2006 22:2020-2027.

doi:10.1186/1471-2164-11-115

Cite this article as: Barrantes et al: Transcriptomic changes arising during light-induced sporulation in Physarum polycephalum. BMC Genomics 2010 11:115.

Submit your next manuscript to BioMed Central and take full advantage of:

- Convenient online submission

- Thorough peer review

- No space constraints or color figure charges

- Immediate publication on acceptance

- Inclusion in PubMed, CAS, Scopus and Google Scholar

- Research which is freely available for redistribution

Submit your manuscript at www.biomedcentral.com/submit 